CARDIOVASCULAR MEDICINE

\title{
Infective endocarditis in Europe: lessons from the Euro heart survey
}

\author{
P Tornos, B lung, G Permanyer-Miralda, G Baron, F Delahaye, Ch Gohlke-Bärwolf, E G Butchart, \\ P Ravaud, A Vahanian
}

Heart 2005;91:571-575. doi: 10.1136/hrt.2003.032128

See end of article for authors' affiliations .....................

Correspondence to: Dr Pilar Tornos, Servei de Cardiologia. Hospital Vall d'Hebron, P Vall d' Hebron 119-129 Barcelona, 08035 Spain; ptornos@vhebron.net

Accepted 22 April 2004
Objectives: To describe the characteristics, treatment, and outcomes of active infective endocarditis (IE) in Europe.

Design: Prospective survey of medical practices in Europe.

Setting: 92 centres from 25 countries.

Patients: The EHS (Euro heart survey) on valvar heart disease (VHD) enrolled 5001 adult patients between April and July 2001. Of those, 159 had active IE.

Results: 118 patients (74\%) had native IE and 41 (26\%) had prosthetic IE. Mean (SD) age was 57 (16) years. Blood cultures were obtained for 113 patients (71\%) before antibiotic treatment was started. Surgery was performed in $52 \%$ of patients. Reasons for surgery were heart failure in $60 \%$, persistent sepsis in $40 \%$, vegetation size in $48 \%$, or embolism in $18 \%$. Surgery was for implantation of mechanical prosthesis in $63 \%$, bioprosthesis in $21 \%$, aortic homograft in $5 \%$, and valve repair in $11 \%$. In-hospital mortality was $12.6 \%$, being $10.4 \%$ in the medical group and $15.6 \%$ in the surgical group. Among the total population of 5001 patients, only $50 \%$ of those with native VHD had been educated on endocarditis prophylaxis and only 33\% regularly attended dental follow up. Of patients with IE who had had a procedure at risk during the preceding year only $50 \%$ had received adequate prophylaxis.

Conclusions: The EHS on VHD shows that patients with active IE have a high risk profile and often undergo surgery. However, there are deficiencies in obtaining blood cultures and applying prophylaxis. Mortality remains high, which is a justification for the improvement of patient management through education and the implementation of guidelines.
D uring recent years several changes have occurred in the clinical and microbiological presentation of active infective endocarditis (IE) and its management..$^{1-5}$ Guidelines have been proposed in several European countries and in the USA.$^{6-8}$ However, little information is available on the contemporary management of the disease and perhaps more important on the adequacy of evaluation and treatment under the current recommendations.

The EHS (Euro heart survey) programme was initiated by the European Society of Cardiology to provide quantitative information on cardiovascular diseases and their management patterns across Europe. ${ }^{9}$ The purpose of the EHS on valvar heart disease (VHD) was to obtain information on current practices in patients with VHD. ${ }^{10}$

This study aimed at analysing the data taken from the EHS on VHD on patients with active IE, paying special attention to clinical presentation, diagnostic procedures, treatment, and adherence to guidelines.

\section{METHODS}

The EHS on VHD was conducted from April to July 2001 in 92 centres across 25 countries and it enrolled prospectively 5001 adults with moderate or severe native valve disease according to transthoracic echocardiography, with probable or definite IE according to the Duke University criteria, or who had had previous valve intervention. Participating clusters, duration of the survey, criteria for patient selection, and data collection on the EHS on VHD have been described previously. ${ }^{10}$ Case report forms comprised details on diagnostic and treatment modalities. Attending physicians were interviewed for decisions regarding the choice of management and treatment.
The population of the present study comprised hospitalised patients who were considered by the attending physicians to have active IE. Active IE was defined as any episode of definite endocarditis according to the Duke criteria requiring antibiotic treatment during the index hospitalisation. Early prosthetic endocarditis was defined as endocarditis occurring in the first two months after valve surgery and late prosthetic valve endocarditis as endocarditis occurring after this time. Heart failure was defined as the presence of any sign of heart failure during hospitalisation.

\section{Statistical analysis}

Quantitative variables were expressed as mean (SD). Variables were compared by the unpaired Student's $t$ test for quantitative variables and the $\chi^{2}$ or Fisher's test for qualitative variables. Data were analysed with SAS statistical software (release 8.2; SAS Institute Inc, Cary, North Carolina, USA) in the Epidemiology Biostatistics and Clinical Research Department of Bichat Hospital.

\section{RESULTS}

\section{Population}

The EHS on VHD enrolled 5001 adult patients with VHD. Countries were grouped into Western, Mediterranean, Eastern, and Northern Europe. ${ }^{10}$ Active IE was the reason for inclusion of 159 patients who formed the basis of this study. The proportion of patients with IE in relation to the total number of patients with VHD was 3\%, being $4.5 \%$ in

Abbreviations: EHS, Euro heart survey; IE, infective endocarditis; NYHA, New York Heart Association; VHD, valvar heart disease 
Table 1 Clinical characteristics of patients with native valve and prosthetic endocarditis

\begin{tabular}{llll}
\hline & $\begin{array}{l}\text { Native valve } \\
\text { IE (n= 1 18) }\end{array}$ & $\begin{array}{l}\text { Prosthetic valve } \\
\text { IE (n=41) }\end{array}$ & Total (n=159) \\
\hline Heart failure & $55 \%$ & $65 \%$ & $58 \%$ \\
Embolism & $31 \%$ & $17 \%$ & $27 \%$ \\
Fever $>38^{\circ}$ & $89 \%$ & $81 \%$ & $87 \%$ \\
\hline IE, infective endocarditis. & & \\
\hline
\end{tabular}

Northern, $2.8 \%$ in Western, 3.4\% in Eastern, and 2.9\% in Mediterranean Europe $(p=0.34)$. IE occurred on native valves in 118 patients $(74 \%)$ and on prosthetic valves in 41 patients $(26 \%)$.

Of the 92 centres, 23 had patients with IE. During this four month period, the number of patients with IE varied between 1-15 in each centre: three centres had $>10$ patients, nine centres had 5-9 patients, and 11 centres had $<5$ patients with IE.

Among patients with native IE, affected valves were aortic in 56, mitral in 35, multiple in 15 , and tricuspid in four. The site was not stated in eight cases of IE, of which four were associated with pacemaker endocarditis. Among the cases of prosthetic endocarditis, three were early IE and 38 were late IE. Mean (SD) age was 56 (17) years among patients with native IE and 61 (13) in prosthetic IE. The percentage of patients older than 70 years was $26 \%$ overall: $22 \%$ in native IE and $36 \%$ in prosthetic IE. One hundred and eleven patients $(70 \%)$ were men. Ninety four patients were aware of having VHD before the diagnosis of endocarditis-that is, 53 of the 118 patients (45\%) with native VHD and all 41 patients with prosthetic IE.

\section{Clinical characteristics \\ Portal of entry}

A portal of entry was identifiable in $48 \%$ of the patients with native IE and in $41 \%$ of the patients with prosthetic IE. In native IE the portal of entry was considered dental in $17 \%$, digestive in $9 \%$, genitourinary in $2 \%$, cutaneous in $8 \%$, iatrogenic in $5 \%$, and other in $7 \%$. In prosthetic IE the portal of entry was considered to be dental in 3\%, digestive in $5 \%$, genitourinary in $11 \%$, cutaneous in 3\%, iatrogenic in $14 \%$, and other in $5 \%$. Drug addiction was reported in eight patients $(5 \%)$.

\section{Clinical presentation}

Table 1 shows clinical characteristics. Most patients (87\%) had fever. Heart failure was present on admission or developed during hospitalisation in 58\%. Twenty seven per cent had an embolic episode $(31 \%$ with native IE and $17 \%$ with prosthetic IE). Location of embolism was, either singly

Table 2 Microorganisms in patients who had blood cultured before antibiotic treatment

\begin{tabular}{llll}
\hline & $\begin{array}{l}\text { Native valve } \\
\text { IE }(\mathbf{n = 7 7 )}\end{array}$ & $\begin{array}{l}\text { Prosthetic } \\
\text { valve IE (n=27) }\end{array}$ & $\begin{array}{l}\text { Total } \\
(\mathbf{n}=104)\end{array}$ \\
\hline Streptococcus viridans & $14 \%$ & $11 \%$ & $13 \%$ \\
Enterococci & $14 \%$ & $15 \%$ & $14 \%$ \\
Other streptococci & $19 \%$ & $4 \%$ & $15 \%$ \\
Staphylococcus aureus & $34 \%$ & $30 \%$ & $33 \%$ \\
Other microorganisms & $7 \%$ & $18 \%$ & $10 \%$ \\
No microorganism & $12 \%$ & $22 \%$ & $14 \%$ \\
\hline
\end{tabular}

113 patients had blood cultured before antibiotic treatment was started and data concerning the type of microorganism were available for 104 of them.
Table 3 Reasons for surgery in active IE

\begin{tabular}{llll}
\hline & $\begin{array}{l}\text { Native valve } \\
\text { IE }(\mathbf{n}=62)\end{array}$ & $\begin{array}{l}\text { Prosthetic } \\
\text { valve IE (n= 20) }\end{array}$ & $\begin{array}{l}\text { Total } \\
(\mathbf{n}=\mathbf{8 2})\end{array}$ \\
\hline Heart failure & $58 \%$ & $65 \%$ & $60 \%$ \\
Persistent sepsis & $39 \%$ & $45 \%$ & $40 \%$ \\
Embolism & $18 \%$ & $20 \%$ & $18 \%$ \\
Vegetation size* & $54 \%$ & $25 \%$ & $48 \%$ \\
Other & $31 \%$ & $40 \%$ & $33 \%$ \\
\hline
\end{tabular}

Patients may have multiple reasons for surgery.

*Vegetation size was the only reason for surgery in four patients with

native IE and in one patient with prosthetic IE.

or in combination, cerebral in 24 patients, abdominal in two, pulmonary in six, and other in nine.

\section{Investigations}

Blood cultures were obtained before antibiotics were started in 113 patients $(71 \%)$. Table 2 shows the results of the microbiological examinations in patients with native and prosthetic IE. Transoesophageal echocardiography was used in $76 \%$ of both native and prosthetic IE. Abnormal echocardiographic findings, defined as vegetation, abscess, perforation, or prosthetic dysfunction, were observed in $82 \%$ of native IE cases and in $70 \%$ of prosthetic IE. When considering also the presence of a regurgitation grade $\geqslant 2 / 4$, the proportions of abnormal transoesophageal echocardiography were $95 \%$ for native valves and $96 \%$ for prostheses. The reasons for transoesophageal echocardiography were, either singly or in combination, diagnosis of IE in $40 \%$, evaluation of IE in $48 \%$, evaluation of IE when transthoracic echocardiography provided suboptimal data in $52 \%$, evaluation of the mechanism of prosthetic dysfunction in 15\%, systematic in $13 \%$, after an embolic event in $8 \%$, and other reasons in $2 \%$ of patients.

Other examinations performed to search for embolism were brain computed tomography in $24 \%$, abdominal computed tomography in $14 \%$, abdominal echocardiography in $45 \%$, and arteriography in $4 \%$ of patients.

\section{Treatment}

Antibiotic treatment was given to $97 \%$ of patients. One hundred per cent of patients with prosthetic IE and 95\% with native IE received intravenous treatment. Two or more antibiotics were administered to $88 \%$ of the patients. Only

Table 4 Clinical features of patients undergoing medical or surgical treatment

\begin{tabular}{llll}
\hline & $\begin{array}{l}\text { Medical } \\
\text { (n=77) }\end{array}$ & $\begin{array}{l}\text { Surgical } \\
\text { (n=82) }\end{array}$ & p Value \\
\hline Age (years) & $60.4(15.8)$ & $54.6(15.8)$ & 0.02 \\
श1 Co-morbid condition* & $74 \%$ & $49 \%$ & 0.002 \\
NYHA class & & & \\
I & $34.7 \%$ & $28.8 \%$ & 0.22 \\
II & $21.3 \%$ & $12.5 \%$ & \\
III & $30.7 \%$ & $36.2 \%$ & \\
IV & $13.3 \%$ & $22.5 \%$ & \\
Congestive heart failure & $31.2 \%$ & $41.5 \%$ & 0.19 \\
LVEDD (mm) & $54(6)$ & $57(12)$ & 0.13 \\
LVEF (\%) & $59(11)$ & $58(11)$ & 0.49 \\
Prosthetic endocarditis & $28.8 \%$ & $24.4 \%$ & 0.66 \\
\hline
\end{tabular}

Data are mean (SD) or percentage of patients.

*Co-morbid conditions were previous myocardial infarction, carotid atherosclerosis, lower limb atherosclerosis, chronic obstructive pulmonary disease, creatinine $>200 \mu \mathrm{mol} / \mathrm{l}$, neurological dysfunction, and coronary artery disease in $\geqslant 1$ vessel.

LVEDD, left ventricular end diastolic dimension; LVEF, left ventricular ejection fraction; NYHA, New York Heart Association. 


\begin{tabular}{|lll|}
\hline $\begin{array}{l}\text { Table } 5 \\
\text { mitral valve IE }\end{array}$ & $\begin{array}{l}\text { Type of surgery in native aortic and } \\
\text { Aortic } \\
(\mathbf{n}=\mathbf{3 4})\end{array}$ & $\begin{array}{c}\text { Mitral } \\
(\mathbf{n}=\mathbf{1 1})\end{array}$ \\
\hline & 27 & 11 \\
\hline Number of patients & $74 \%$ & $36 \%$ \\
Mechanical prosthesis & $15 \%$ & $36 \%$ \\
Bioprosthesis & $7 \%$ & 0 \\
Homograft & $4 \%$ & $28 \%$ \\
Valve repair & & \\
\hline
\end{tabular}

five patients with native IE were treated orally. Mean duration of antibiotic treatment was 30 days and 25\% of the patients were treated for more than 45 days.

Of the 159 patients with IE, $82(52 \%)$ underwent surgery: 62 patients with native IE (53\%) and 20 patients with prosthetic IE (49\%). Surgery was performed in $61 \%$ of patients with native aortic IE, $31 \%$ with mitral IE, $80 \%$ with multiple valve IE, and $49 \%$ with prosthetic IE.

Surgery was performed within the first 24 hours in $12.5 \%$, during the initial hospitalisation in $75 \%$, and electively in $12.5 \%$.

Table 3 lists the reasons for surgery in both native and prosthetic valve IE. Most patients had more than one reason for surgical intervention. Vegetation size was the only indication for surgery in $6 \%$ of patients.

Patients who were treated medically were significantly older and had more co-morbidity than those who were treated surgically. On the other hand, there was a trend towards a higher incidence of New York Heart Association (NYHA) class IV and congestive heart failure among patients who underwent surgery (table 4).

Of the 45 patients who underwent surgery for left sided single valve IE, surgical techniques were valve replacement with a mechanical prosthesis in $63 \%$ and a bioprosthesis in $21 \%$, homografts in $5 \%$, and valve repair in $11 \%$ (table 5 ). Among the 20 patients whose aortic valve was replaced with a mechanical prosthesis for native aortic IE, the most frequent reasons for using a mechanical prosthesis were, either singly or in combination, young age in $90 \%$, physician's preference in $75 \%$, other indication for treatment with an anticoagulant in $15 \%$, and renal failure in $10 \%$. Homograft unavailability was considered the reason for using a bioprosthesis in only two patients $(50 \%)$ of those receiving a bioprosthesis. In the remaining patients in whom bioprosthesis or mechanical valves were used the reasons for not using homografts were not given.

Of the patients who had heart failure complicated IE, 36\% were not operated on during the active phase.

\section{Outcome}

At 30 days 20 patients had died, accounting for $12.6 \%$ of the whole series (table 6). The cause of death was haemodynamic in five patients, sepsis in six patients, neurological in one patient, postoperative in three patients, and other reasons in

Table 6 Thirty day mortality in patients treated medically or surgically for IE

\begin{tabular}{llll}
\hline & $\begin{array}{l}\text { Medical } \\
(\mathbf{n}=\mathbf{7 7})\end{array}$ & $\begin{array}{l}\text { Surgical } \\
(\mathbf{n}=\mathbf{8 2})\end{array}$ & $\begin{array}{l}\text { Total } \\
(\mathbf{n}=159)\end{array}$ \\
\hline $\begin{array}{l}\text { Native valve IE }(\mathrm{n}=118) \\
\text { Prosthetic valve IE }(\mathrm{n}=41)\end{array}$ & $8.9 \%$ & $14.5 \%$ & $11.9 \%$ \\
Total $(\mathrm{n}=159)$ & $14.3 \%$ & $15.0 \%$ & $14.6 \%$ \\
\hline
\end{tabular}

five patients. Mortality among the patients treated medically was $10.4 \%$ and $15.6 \%$ among the patients who underwent surgery $(\mathrm{p}=0.42)$.

\section{Prophylaxis}

Information on endocarditis prophylaxis was obtained from the total population of 5001 patients with valve disease. Fifty per cent of the patients with native valve disease stated that they had received education on prophylaxis and 33\% that they had regular follow up visits with a dentist. These figures were $70 \%$ and $47 \%$ for the patients who had previous conservative valve surgery, and $77 \%$ and $53 \%$ for patients with prosthetic valves.

Among the 159 patients who had IE, 94 had VHD previously diagnosed. Of them, 42 had undergone a procedure at risk in the year before endocarditis and only $21(50 \%)$ had received antibiotic prophylaxis.

\section{DISCUSSION}

The EHS on VHD provides a unique opportunity both to analyse the current contemporary management and outcomes of IE in Europe and to assess the rationale for the management of IE. Even if the design of the EHS does not allow for a precise assessment of the prevalence of the disease, the present findings show that active endocarditis is not a common disease: patients with endocarditis account for only $3.2 \%$ of the total population in the EHS on VHD. These findings are consistent with the incidence of IE, which was estimated at only 31 cases per million adults in a recent French survey. ${ }^{1}$

The clinical and microbiological presentation observed suggests that IE tends to be a particularly severe disease today. Patients with endocarditis are often elderly. ${ }^{1-5}{ }^{11}$ In the EHS, $26 \%$ of patients were older than 70 years. In the registry by Delahaye and colleagues, ${ }^{5} 33 \%$ of patients were older than 67 years. In the French surveys, the incidence of endocarditis increased between 1991 and 1999 among patients older than 50 years and peaked at 145 cases per million men between 70 and 80 years old. ${ }^{1}$ The severity of the disease is also shown by the high incidence of native aortic and prosthetic valve endocarditis. The decreasing proportion of infections with Streptococcus viridans and the increasing proportions of Staphylococcus aureus and enterococcal disease are consistent with recent recently published data. ${ }^{12}$ Lastly, of the 118 patients with native valve IE, $45 \%$ were aware of having VHD, which is consistent with rates of $51 \%$ and $53 \%$ in two other recent series. ${ }^{15}$

All patients had had transthoracic echocardiography since this was mandatory for inclusion in the EHS on VHD. The use of transoesophageal echocardiography was $76 \%$ in the total series and the reasons given for its performance are in accordance with the guidelines. ${ }^{6}$ However, in patients with prosthetic valves the use of transthoracic echocardiography should probably be higher. ${ }^{13}$ In the recent French survey, 90\% of the patients underwent both transthoracic and transoesophageal echocardiography, and $10 \%$ had only transthoracic echocardiography. ${ }^{1}$

Radiological examination was not often used to search for embolic events. In particular, computed tomography was used for only a quarter of the patients. It has been shown that the systematic performance of computed tomography in IE may show asymptomatic embolic events in as many as $21 \%$ of the patients with IE. ${ }^{14}$ This may have implications for indications for surgery but the impact of systematic radiological examinations in IE to search for embolism has not been studied so far.

In the present survey, most patients received intravenous antibiotics for one month or more. A disappointing finding was that as many as $29 \%$ of patients did not have blood 
cultures taken before antibiotics were started. A consistent observation was made in a previous registry in France, ${ }^{5}$ where $58 \%$ of the patients who had known VHD and fever did not have blood cultures before antibiotic treatment. Another area of concern is the high incidence of negative blood cultures (14\%). Our study cannot answer whether this percentage reflects previous antibiotic use, endocarditis caused by microorganisms that require serological testing for diagnosis, or deficiencies in the microbiological examination. In recent large observational series, the proportion of negative blood cultures ranged from 9-14\%. ${ }^{124}$ The proportion of patients with negative blood cultures is likely to be higher in such a survey than in studies involving selected centres.

In the EHS, $52 \%$ of the patients underwent surgery, which was performed on an emergent or urgent basis in most patients. This is at least as high as in the most recent French survey $(49 \%)$ and significantly higher than in previous studies. ${ }^{1}$

The adequacy of indications for surgery with the guidelines should be evaluated with particular caution. When surgery was performed, indications were often in accordance with the current recommendations, heart failure being the most common, followed by persistent infection and embolism. ${ }^{6-8}$ In patients with native IE, vegetation size was cited as an indication for surgery in $54 \%$ of the cases, which may seem particularly high because there is no consensus for such an indication. However, there often were multiple indications and vegetation size was the sole indication for surgery in only $6.5 \%$ of patients with native IE. Conversely, an unexpectedly high proportion of patients had at least transient congestive heart failure and were not operated on. Older age and comorbidity may partly explain the lack of surgical indication in these patients. Delahaye and colleagues ${ }^{5}$ also often found an absence of indication for surgery despite the presence of heart failure.

The type of surgery performed was mainly valve replacement with either mechanical or biological prostheses. Although homograft is claimed to be the ideal valve substitute in aortic endocarditis complicated with abscess ${ }^{15} 16$ its use was only $7 \%$. This may reflect the small number of patients with aortic abscesses, the limited availability of homografts, or a limited adoption of this recommendation in real life. It is of interest to note that the physician's (or surgeon's) preference was the stated reason in up to $75 \%$ of the patients in whom a mechanical prosthesis was used as a valve substitute during IE. The valve was repaired in $28 \%$ of patients with native mitral IE, which is a relatively high figure for an international survey. Good results from valve repair have been reported in certain surgical series but the experience of mitral valve repair during the acute phase of IE is limited and its use is subject to debate. ${ }^{17-19}$

The in-hospital mortality (12.6\%) was high in this series. However, the fatality in EHS compares favourably with that of the most recent registry, ${ }^{1}$ which found a $16.6 \%$ mortality. There was also an unusual trend towards a lower mortality among medically treated patients. These estimates should, however, be interpreted with caution given the limited number of patients. Although patients who underwent surgery were younger and had less co-morbidity, more of them were in NYHA class IV and had diagnosed congestive heart failure, which are both strong predictors of operative mortality in IE.

Even if the role of antibiotic prophylaxis in the prevention of endocarditis is subject to debate, the level of education on endocarditis prophylaxis found by the EHS is inadequate and current guidelines on prophylaxis are not being followed..$^{20}$ Only half of the patients with native valve disease claimed to be educated about prophylaxis and one third regularly attended dental follow up. These numbers were only slightly better among patients with prosthetic valves. Such unsatisfactory figures have also been found in other national registries. In a recent survey in Germany only $55 \%$ of patients with VHD knew about the need for dental prophylaxis, ${ }^{22}$ and in the study by Delahaye and colleagues in France ${ }^{5}$ eight of 11 patients with an iatrogenic portal of entry necessitating antibiotic prophylaxis did not receive it. This stresses that every effort is needed to improve the education of both patients with known valvar disease and their physicians.

However, an inherent limitation of the effectiveness of education is related to the high percentage of endocarditis occurring in patients without previously diagnosed heart disease.

\section{Study limitations}

Participating centres were not selected at random; therefore, a selection bias cannot be ruled out. Nevertheless, a wide range of both university and non-academic hospitals across a large number of countries participated. The number and the diversity of participating centres is a distinctive feature of the EHS. This may account for certain findings such as the high percentage of negative blood cultures and the low proportion of early prosthetic IE relative to series on IE, which usually originate from tertiary centres with a particular expertise. The fact that only four of the 25 centres accounted for one third of the present study population is a good illustration of the trend towards concentrating the treatment of IE in specialised centres. In accordance with the objectives of the EHS, these findings provide an insight on current practices in the "real world". This is of particular interest regarding IE as it is not a common disease and is not always managed by highly experienced teams.

The population of the present study reflects the management of patients who were considered by the attending physician to have endocarditis and this was consistent with the pragmatic evaluative approach of the EHS. The recommendation was to enrol patients with definite IE according to the Duke criteria but it was not possible to reclassify or exclude patients who would not have met criteria for definite IE, in particular as regards patients with negative blood cultures. In cases that may have not been classified as definite according to the Duke criteria, the diagnosis of IE may be based mainly on clinical judgment. The sensitivity of the Duke criteria has been evaluated at about $80 \%$ for definite IE; therefore, the absence of criteria for definite endocarditis does not firmly exclude the diagnosis of IE. ${ }^{23} 24$

Lastly, the rather limited number of patients does not allow for accurate conclusions in some specific subgroups.

In summary, the EHS on VHD provides unique contemporary data on the presentation, management, and outcomes of active IE in Europe. The types of endocarditis reflect the changes towards severe forms of the disease in elderly patients, with frequent involvement of the aortic valve and a high frequency of staphylococcal and enterococcal infections. Surgery is often performed during active IE but it seems still to be underused in patients with heart failure. In most patients who undergo surgery valves are replaced by mechanical prostheses although new surgical techniques are beginning to be used. IE still carries a poor prognosis, even if the outcomes in EHS on VHD compare favourably with recent reports. Education on the use of prophylaxis with known valvar disease should be improved for both patients and physicians, as well as highlighting to physicians the need for performance of blood cultures before antibiotic treatment. Overall these findings reflect the fact that IE is still a disease with a poor prognosis that requires a continuing effort in the implementation of existing guidelines and education of the patients to improve outcome. 


\section{ACKNOWLEDGEMENTS}

The Euro heart survey on valvar heart disease was funded by the European Society of Cardiology, the Dutch Heart Foundation, the Fédération Française de Cardiologie/Société Française de Cardiologie, the Hellenic Cardiological Society, the Swedish Heart and Lung Foundation, the European Commission Grant (Infermed/Mansev Project), and the Toray Medical Company.

\section{Authors' affiliations \\ P Tornos, G Permanyer-Miralda, Hospital Vall d'Hebron, Barcelona, Spain \\ B lung, G Baron, P Ravaud, A Vahanian, Bichat Hospital, Paris, France F Delahaye, Hopital Cardiologique, Lyons, France \\ Ch Gohlke-Bärwolf, Heart Centre, Bad Krozingen, Germany \\ E G Butchart, University Hospital, Cardiff, UK}

\section{REFERENCES}

1 Hoen B, Alla F, Selton-Suty Ch, et al. Changing profile of infective endocarditis: results of a 1-year survey in France. JAMA 2002;288:75-81.

2 Tornos MP, Olona M, Permanyer-Miralda G, et al. Is the clinical spectrum and prognosis of infective endocarditis in non-addicts changing? Eur Heart $J$ 1995; 16:1686-91.

3 Hogevik H, Olaison L, Andersson R, et al. Epidemiologic aspects of infective endocarditis in an urban population: a 5 -year prospective study. Medicine 1995; 74:324-39.

4 Castillo JC, Anguita MP, Ramirez A, et al. Long-term outcome of infective endocarditis in patients who were not drug addicts: a 10-year study. Heart 2000;83:525-30.

5 Delahaye F, Rial MO, de Gevigney G, et al. A critical appraisal of the quality of the management of infective endocarditis. J Am Coll Cardiol 1999;33:788-93.

6 Bonow RO, Carabello B, Deleon AC, et al. ACC/AHA guidelines for the management of patients with valvular heart disease J Am Coll Cardiol 1998; 32: 1486-588

7 Horstkotte D, Follath F, Gutschik E, et al. Guidelines on prevention, diagnosis and treatment of infective endocarditis. The task force on infective endocarditis of the European Society of Cardiology. Eur Heart J 2004;25:267-76.

8 Azpitarte J, Alonso A, Garcia Gallego F, et al. Guias de prectica clinica de la Sociedad Española de Cardiologia en valvulopatias. Rev Esp Cardiol 2000;53:1209-78.
9 Boersma E, Manini M, Wood DA, et al. Cardiovascular diseases in Europe. Euro heart survey and national registries of cardiovascular diseases and patient management. Sophia Antipolis: European Society of Cardiology, 2002.

10 lung B, Baron G, Butchart E, et al. A prospective survey of patients with valvular heart disease in Europe: the Euro heart survey on valvular heart disease. Eur Heart J 2003;24:1231-43.

11 Werner GS, Schulz R, Fuchs JB, et al. Infective endocarditis in the elderly in the era of transesophageal echocardiography: clinical features and prognosis compared with younger patients. Am J Med 1996;100:90-7.

12 Roder BL, Wandall DA, Frimodt-Moller N, et al. Clinical features of Staphylococcus aureus endocarditis: a 10 year experience in Denmark. Arch Intern Med 1999; 159:462-69.

13 Li JS, Sexton DJ, Mick N, et al. Proposed modifications for the Duke criteria for the diagnosis of infective endocarditis. Clin Infect Dis 2000;30:633-8.

14 Di Salvo G, Habib G, Pergola V, et al. Echocardiography predicts embolic events in infective endocarditis. J Am Coll Cardiol 2001;37:1069-76.

15 Kirklin JK, Kirklin JW, Pacifico AD. Aortic valve endocarditis with aortic root abscess cavity: surgical treatment with aortic valve homograft. Ann Thorac Surg 1988;45:674-7.

16 Knosalla C, Weng Y, Yankah AC, et al. Surgical treatment of active infective aortic valve endocarditis with associated periannular abscess. 11-year results. Eur Heart J 2000;21:490-7.

17 Dreyfus G, Serraf A, Jebara VA, et al. Valve repair in acute endocarditis. Ann Thorac Surg 1990;49:706-13.

18 Pagani FD, Monaghan HL, Deeb GM, et al. Mitral valve reconstruction for active and healed endocarditis. Circulation 1996;94:11133-8.

19 Muehrcke DD, Cosgrove DM 3rd, Lytle BW, et al. s there an advantage to repairing infected mitral valves? Ann Thorac Surg 1997;63:1718-24.

20 Leport C, Horstkotte D, Burckhardt D, et al. Antibiotic prophylaxis from an international group of experts towards a European consensus. Group of Experts of the International Society for Chemotherapy. Eur Heart $J$ 1995;16(suppl B):126-31

21 Dajani AS, Taubert KA, Wilson W, et al. Prevention of bacterial endocarditis: recommendations by the American Heart Association. JAMA 1997;277:1794-801.

22 Gohlke-Bärwolf C, Rost $M$, Roskamm H. Prevention of bacterial endocarditis in Germany. Eur Heart J 1995; 16(suppl B): 1 10-3.

23 Habib G, Derumeaux G, Avierinos JF, et al. Value and limitations of the Duke criteria for the diagnosis of infective endocarditis. J Am Coll Cardiol 1999;33:2023-9.

24 Cecchi E, Parrini I, Chinaglia A, et al. New diagnostic criteria for infective endocarditis: a study of sensitivity and specificity. Eur Heart $J$ 1997; 18:1149-56

\section{ELECTRONIC PAGES}

\section{Heart Online case reports: www.heartjnl.com}

7

he following electronic only articles are published in conjunction with this issue of Heart.

\section{Gentamicin induced ototoxicity during treatment of enterococcal endocarditis: resolution with substitution by netilmicin}

J E East, J E Foweraker, F D Murgatroyd

Enterococcal endocarditis can be very difficult to eradicate, requiring prolonged treatment with a combination of a penicillin and an aminoglycoside. In this patient with a pacemaker associated enterococcal endocarditis, ototoxicity occurred due to total gentamicin dose despite plasma concentrations consistently within the treatment range. Substitution with netilmicin, without a break in aminoglycoside treatment, resulted in a rapid improvement in hearing and allowed the required course of aminoglycoside to be completed. The risk factors for ototoxicity with gentamicin are reviewed, in particular the dangers of increasing age and of multiple and prolonged courses. Close treatment monitoring does not totally avoid this risk, especially when prolonged aminoglycoside treatment is required. This case emphasises the need for prompt investigation and adequate, definitive treatment of enterococcal endocarditis to avoid the increased risk consequent on repeated courses of antibiotics. The resolution of the ototoxicity with netilmicin is consistent with other reports of lower cochleotoxicity than with other aminoglycosides.

(Heart 2005;91:e32) www.heartjnl.com/cgi/content/full/91/ $5 / \mathrm{e} 32$

\section{Lysyl oxidase deficiency: a new cause of human arterial dissection}

I Sibon, P Sommer, J M Daniel Lamaziere, J Bonnet Spontaneous coronary artery dissection is a rare cause of myocardial ischaemia. The underlying mechanism is unknown but some dissections are associated with extracellular matrix disorganisation of genetic origin. A deficiency in extracellular matrix protein cross links has rarely been studied. A single clinical case of spontaneous coronary artery dissection is reported. Lysyl oxidase (LOX) and extracellular matrix organisation were investigated by skin immunohistology and polymerase chain reaction of LOX expression. Both approaches found a dramatic LOX decrease. LOX deficiency has a major role in human arterial wall organisation during development. The suspected mechanism is an elastin or collagen polymer cross linking deficiency.

(Heart 2005;91:e33) www.heartjnl.com/cgi/content/full/91/ $5 / \mathrm{e} 33$ 